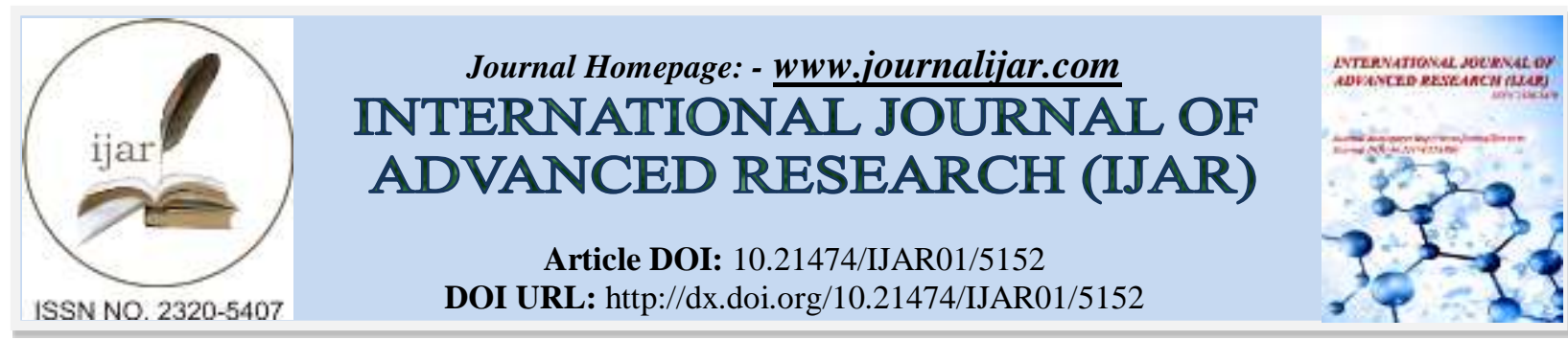

RESEARCH ARTICLE

\title{
RARE OPHTHALMIC MANIFESTATIONS OF RICKETTSIAL INFECTIONS.
}

\author{
Seena Sankar ${ }^{1}$, Shruthi Kulkarni ${ }^{1}$, Manjoo CS $^{2}$ and Nitin Yashas Murthy ${ }^{1}$. \\ 1. Dept. of General Medicine, St. John's Medical College, Bangalore-56034. \\ 2. Dept. of Ophthalmology, St. John's Medical College, Bangalore-560034.
}

\section{Manuscript Info}

Manuscript History

Received: 12 June 2017

Final Accepted: 14 July 2017

Published: August 2017

Key words:-

Anterior uveitis, Rickettsial fever, typhus, ocular manifestations, infectious uveitis.

\begin{abstract}
Background: Rickettsial infections are re-emerging health problems in the country. There is a paucity of data on the ophthalmic manifestations of rickettsial disease in India. Majority of the ocular manifestations reported involve the posterior segment with very few documented cases of anterior segment manifestations. We report cases of febrile illness associated with acute anterior uveitis which were diagnosed as rickettsial infection.

Findings: Four patients presented with febrile illness with acute anterior uveitis. Three out of the four patients developed eye signs between day eleven to day thirteen of onset of fever. Three patients had only unilateral uveitis with the fourth case having bilateral. Patients were treated with doxycycline along with steroid and cycloplegic eye drops and improved symptomatically. No recurrence of anterior uveitis was noted on follow up.

Conclusion: The possibility of rickettsial fever must be considered in the differential diagnosis of acute febrile illness with acute anterior uveitis as early initiation of treatment can lead to better outcomes.
\end{abstract}

Copy Right, IJAR, 2017,. All rights reserved.

\section{Introduction:-}

Rickettsial diseases are a group of diseases caused by obligate intracellular gram negative bacteria. The mode of transmission to human beings is by the bite of arthropods such as ticks. Rickettsial agents are classified into three major categories: the spotted fever group, the typhus group, and the scrub typhus $(1,2)$. Ocular involvement is common in patients with rickettsioses, but since it is frequently asymptomatic and self-limited, it may be easily overlooked. There are plenty of reports which document ocular manifestations of rickettsial infections worldwide (3, 4). But these highlight mainly the posterior segment involvement as the most common ocular manifestations in rickettsial disease are retinitis, retinal vascular involvement and optic disc changes $(5,6)$. There are very few reports on the anterior segment involvement in rickettsial disease $(7,8)$. Furthermore Indian studies have predominantly looked at the systemic manifestations of rickettsial infections with a paucity of data on ocular manifestations $(9,10)$.

This paper attempts to highlight that the occurrence of acute anterior uveitis in the setting of fever should prompt for investigation and treatment of rickettsial disease. The value of Weil Felix test, though not a gold standard has been proven in Indian studies for the diagnosis of rickettsiois $(11,12)$. Weil Felix titers of more than 1:160 for OX K and more than 1:80 for OX 2 and OX 19 were considered significant for diagnosis (11). 
We present a series of four cases of acute anterior uveitis in the presence of febrile illness diagnosed as rickettsial infection.

\section{Findings:-}

\section{Case 1:-}

45 year old male with no known comorbidities presented with history of fever for 7 days. Initial evaluation was suggestive of thrombocytopenia with transaminitis. Patient was empirically started on ceftriaxone. Fever work up sent for Quantitative buffy coat (QBC) of malaria, dengue serology were negative. Widal and Weil Felix test (titres $<1: 20$ ) were negative. Blood and urine cultures showed no growth. On Day 10 of fever, Patient developed erythematous itchy rash over trunk, back and extremities and on examination was found to have few discrete papules on bilateral upper limbs and few erythematous spots on soles and palms with petechiae in oral mucosa. Patient was empirically started on Doxycycline in view of possibility of rickettsial rash. On Day 11 of fe ver, patient developed left eye pain and redness. Ophthalmological examination was suggestive of congested anterior segment with presence of aqueous cells 2+ in left eye with fine Keratin Precipitates (KPs) suggestive of acute anterior uveitis. There was no evidence of posterior uveitis or retinal vasculitis or granulomatous uveitis. Repeat Weil Felix test demonstrated increase in titres against OX-2 of more than 1:320. Doxycycline was continued for total of 7 days. Patient improved symptomatically with Doxycycline and Steroid and cycloplegic eye drops. Repeat Ophthalmological examination done showed resolution of the anterior uveitis.

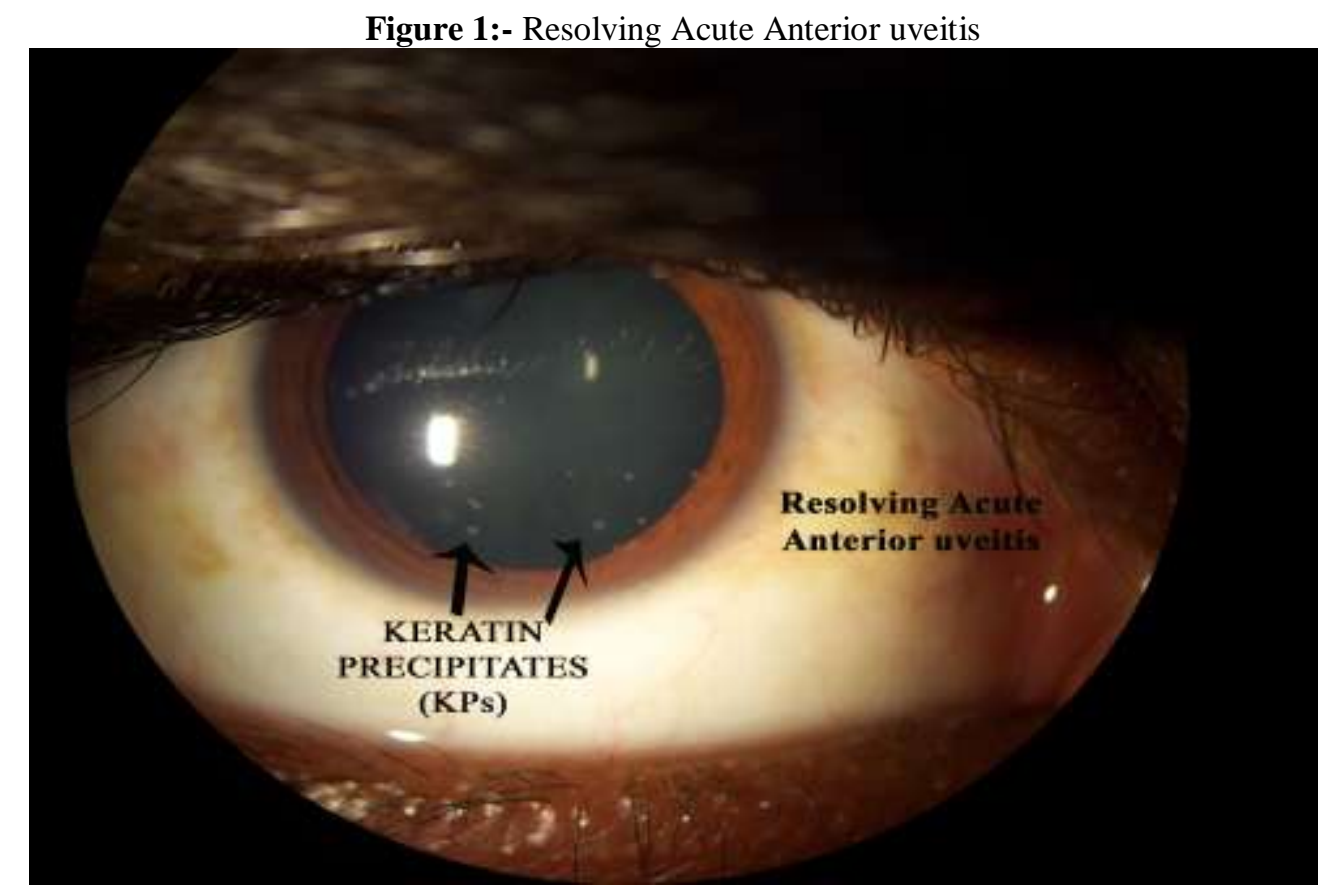

Case 2:-

47 year old male with no known comorbidities presented with history of fever for 7 days along with vomiting for 5 days and was admitted in the ICU with tachycardia, tachypnea and hypotension. Initial investigations were suggestive of thrombocytopenia with transaminitis and increased creatinine. Patient was empirically started on ceftriaxone. Hypotension resolved with fluid support. In view of tachypnea, patient was maintained on NIV (noninvasive ventilation). Initial evaluation of fever done in ICU was negative for QBC Malaria, Dengue serology and Widal test. Blood culture showed no growth and Chest X-ray was normal. Patient was transferred out of the ICU after stabilisation. In view of persisting fever spikes, patient was empirically started on Doxycycline on Day 11 of fever. On Day 12 of fever, patient developed redness of both eyes with photophobia. Ophthalmological examination was suggestive of congestion of anterior segment bilaterally with occasional cells and no Keratin precipitates (KPs). There was no evidence of posterior uveitis or retinal vasculitis or granulomatous uveitis. Hence a diagnosis of bilateral acute anterior uveitis was made. Weil Felix test was sent on Day 12 and demonstrated titres of 1:160 against OX-K. Doxycycline was continued for a total of 7 days. Patient symptomatically improved with resolution of thrombocytopenia, transaminitis and renal functions. Ophthalmological resolution was obtained with treatment consisting of steroid eye drops and cycloplegic eye drops. 


\section{Case 3:-}

37 year old male with history of bronchial asthma presented with history of fever for 10 days. Clinical examination was unremarkable. Investigations was suggestive of thrombocytopenia and mild transaminitis. Investigations done two days prior to admission were negative for QBC Malaria, Dengue serology. Widal and Weil Felix tests (titres $<1: 20)$ were negative. Blood and urine cultures showed no growth. Patient was empirically started on ceftriaxone. Three days after admission, on day 13 of fever, patient developed redness and pain in right eye with photophobia. Ophthalmological examination was suggestive of anterior segment congestion in right eyes with no KPs and 2+Cells with no evidence of posterior uveitis or retinal vasculitis or granulomatous uveitis. Doxycycline was started empirically. Repeat Weil Felix sent on Day 14 of fever was positive for OX2 1:320 and Ox19 1:160. Patient improved symptomatically and had complete ophthalmological resolution after treatment with steroid eye drops and cycloplegic eye drops.

\section{Case 4:-}

24 year old female with no known comorbidities presented with history of low grade fever on and off for one month with history of being treated with cephalosporin and amoxicillin. No history of weight loss. Prior investigations for evaluation of fever at the native place of the patient was negative for QBC malaria, dengue serology and Widal. Clinical examination was unremarkable. Initial investigations of haemogram, biochemical parameters, urine routine and microscopy, Chest X-ray, ultrasound abdomen and pelvis were normal. Patient was afebrile since admission. In view of history of headache, patient underwent neuroimaging and CSF analysis which was normal. Further serological work up were planned if patient developed fever. After 2 days of stay in hospital, patient developed sudden onset of redness and pain in left eye associated with headache and photophobia. Ophthalmological examination was suggestive of acute anterior uveitis with congested anterior segment and 1+ cells in anterior chamber along with fine KPs with no evidence of retinal vasculitis or posterior uveitis or granulomatous uveitis. In view of history of fever with anterior uveitis, Weil Felix was sent which was positive (OX2 titres 1:160). Patient was started on Doxycycline along with symptomatic treatment with steroid eye drops and cycloplegic eye drops. Symptomatically improved and was discharged.

All these patients are on regular follow up on outpatient basis and did not have any recurrence of anterior uveitis .

Figure 2:- Slit lamp examination of Acute Anterior Uveitis

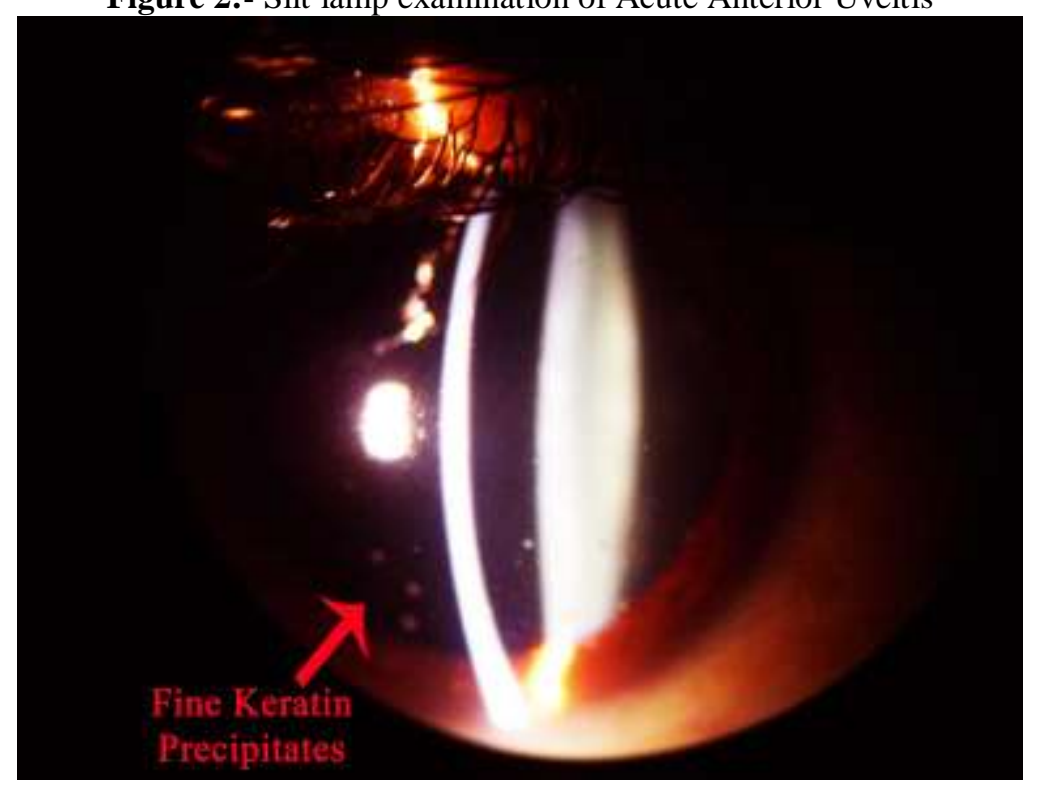


Table 1:- Characteristics of Fever, onset of eye symptoms, Weil Felix titres

\begin{tabular}{|l|l|l|l|l|}
\hline Patients & $\begin{array}{l}\text { Duration of fever } \\
\text { before admission }\end{array}$ & $\begin{array}{l}\text { Day of onset of eye } \\
\text { symptoms }\end{array}$ & $\begin{array}{l}\text { Day of Weil felix } \\
\text { positivity }\end{array}$ & $\begin{array}{l}\text { Presence of four fold } \\
\text { rise in titres }\end{array}$ \\
\hline 1 & 7 days & Day 11 of fever & Day 12 of fever & Yes \\
\hline 2 & 7 days & Day 12 of fever & Day 12 of fever & N/A \\
\hline 3 & 10 days & Day 13 of fever & Day 14 of fever & Yes \\
\hline 4 & 1 month & Day 32 of fever & Day 33 of fever & N/A \\
\hline
\end{tabular}

Table 2:- Ophthalmological findings of anterior uveitis

\begin{tabular}{|l|l|l|l|}
\hline Patients & Eye involvement & Characteristic & Posterior chamber and fundus \\
\hline 1. & Left eye & 2+ cells, $1+$ flare, fine KPs+ & Normal \\
\hline 2. & Bilateral & Occasional cells, 1+ flare & Normal \\
\hline 3. & Right eye & 2+ cells, $1+$ flare & Normal \\
\hline 4. & Left eye & $1+$ cells, $1+$ flare, fine and pigment KPs+ & Normal \\
\hline
\end{tabular}

\section{Discussion:-}

We have described 4 cases of rickettsial infection along with the manifestation of anterior uveitis. We considered the diagnosis of Rickettsial infection when the Weil Felix titres were more than 1:160 or a fourfold rise in titres could also be demonstrated. This is in accordance with a study carried out in Karnataka where WFT titers more than 1:160 for OX K and more than 1:80 for OX 2 and OX 19 were considered significant for diagnois (11). Based on the serological profile of the weil felix text, in our study we have diagnosed 1 case of scrub typhus, 1 case of Indian tick typhus ( spotted fever) and 2 cases with markedly raised tires against OX2 ( unclassified). In a similar Indian study of rickettsial infection with ocular manifestation which predominantly highlighted the posterior segment manifestations, 5 cases were classified as ambiguous, four cases were of Indian Tick typhus and one case was Epidemic/Endemic typhus (13).

While one of the first papers appeared in 1969 to describe anterior non granulomatous uveitis as part of rickettsial fever (7), there have been very few case reports documenting the anterior segment manifestations in rickettsial fever $(7,8,14)$. A spurt of tick blood causing contamination of the conjunctiva has been postulated as a potential source of entry for R.Rickettsii as well as R.conorri infection $(15,16)$. Various other adnexal and anterior segment manifestations have been described in association with rickettsial fever such as conjunctival petechiae, subconjunctival hemorrhage, keratitis, non granulomatous anterior uveitis (17).

3 of our 4 cases had unilateral eye involvement while 1 case had bilateral. The number of cells in the anterior segment ranged from occasional to 2+. Most of the case reports on anterior segment manifestations have documented bilateral involvement $(8,14)$. It was noted in our study that a majority of the eye manifestations developed in the second week of the onset of fever which is in accordance with another Indian study where the presentation of eye manifestations were between the second and fourth week of fever (13).

\section{Conclusion:-}

It is imperative to maintain a high degree of suspicion of Rickettsial infection in a patient with acute febrile illness presenting with acute anterior uveitis. Furthermore, being aware of this rare ocular manifestation of rickettsial fever would help in early diagnosis and initiation of systemic therapy with doxycycline. Recognition of the ocular manifestation by the physician would also prompt timely referral as early treatment of ocular inflammation can lead to good visual prognosis. We would also like to suggest to repeat Weil Felix test for a second time even if the first test was negative, in the cases of febrile illness with acute anterior uveitis as we noted significant titres only towards the end of second week.

\section{References:-}

1. Parola P, Raoult D (1998) Rickettsioses eruptives. EMC (Elsevier Paris) Maladies infectieuses, 8-037-I-20, 24 p.

2. Mc Dade JE (1998) Rickettsial diseases. In: Hausler WJ, Sussman M, eds. Topley and Wilson's Microbilogy and Microbial Infections, 9th ed. London: Arnold 3:995-1011.

3. Alio, J., Ruiz-Beltran, R., Herrero-Herrero, J. I., Hernandez, E., Guinaldo, V., \& Millan, A. (1987). Retinal manifestations of Mediterranean spotted fever. Ophthalmologica, 195(1), 31-37. 
4. Khairallah, M., Ladjimi, A., Chakroun, M., Messaoud, R., Yahia, S. B., Zaouali, Romdhane, F.B \& Bouzouaia, N. (2004). Posterior segment manifestations of Rickettsia conorii infection. Ophthalmology, 111(3), $529-534$.

5. Agahan, A. L., Torres, J., Fuentes-Páez, G., Martínez-Osorio, H., Orduña, A., \& Calonge, M. (2011). Intraocular inflammation as the main manifestation of Rickettsia conorii infection. Clinical ophthalmology (Auckland, NZ), 5, 1401.

6. Abroug, N., Khochtali, S., Kahloun, R., Mahmoud, A., Attia, S., \& Khairallah, M. (2014). Ocular Manifestations of Rickettsial Disease. Journal of Infectious Diseases and Therapy.

7. Cherubini, T. D., \& Spaeth, G. L. (1969). Anterior Nongranulomatous Uveitis Associated With Rocky Mountain Spotted Fever: First Report of a Case. Archives of Ophthalmology, 81(3), 363-365.

8. Beltran, L. M., García, S., Vallejo, A. J., \& Bernabeu-Wittel, M. (2011). Bilateral anterior uveitis and Rickettsia typhi infection. Enfermedades infecciosas y microbiologia clinica, 29(3), 235.

9. Mittal, V., Gupta, N., Bhattacharya, D., Kumar, K., Ichhpujani, R. L., Singh, S., Chhabra, M.\& Rana, U. V. S. (2012). Serological evidence of rickettsial infections in Delhi. The Indian journal of medical research, 135(4), 538.

10. Dasari, V., Kaur, P., \& Murhekar, M. V. (2014). Rickettsial disease outbreaks in India: A review. Annals of Tropical Medicine and Public Health, 7(6), 249.

11. Ajantha, G. S., Patil, S. S., Chitharagi, V. B., \& Kulkarni, R. D. (2013). Rickettsiosis: a cause of acute febrile illness and value of Weil-Felix test. Indian journal of public health, 57(3), 182.

12. Mahajan, S. K., Kashyap, R., Kanga, A., Sharma, V., Prasher, B. S., \& Pal, L. S. (2006). Relevance of WeilFelix test in diagnosis of scrub typhus in India. JAPI, 54, 619.

13. Kawali, A., Mahendradas, P., Srinivasan, P., Yadav, N. K., Avadhani, K., Gupta, K., \& Shetty, R. (2015). Rickettsial retinitis - an Indian perspective. Journal of ophthalmic inflammation and infection, 5(1), 37.

14. Pradeep, T. G., Satyanarayanan, S., \& Krishnaswamy, M. (2015). Bilateral Uveitis in Rickettsial Disease. Journal of Infectious Diseases and Therapy, 1-2.

15. Bazin, R. (2002). Rickettsial diseases. Diagnosis and treatment of uveitis. Philadelphia, PA: WB Saunders Co, 297-304.

16. Pinna, A., Sotgiu, M., Carta, F., Zanetti, S., \& Fadda, G. (1997). Oculoglandular syndrome in Mediterranean spotted fever acquired through the eye. British journal of ophthalmology, 81(2), 168-168.

17. Khairallah, M., Jelliti, B., \& Jenzeri, S. (2009). Emergent infectious uveitis. Middle East African journal of ophthalmology, 16(4), 225. 